\title{
Successful removal of side branch-jailed stent struts by reused balloon
}

\author{
Kazumasa Kurogi ${ }^{1}$, Kenji Sakamoto ${ }^{2}$, Kyohei Marume ${ }^{1}$, Hiroaki Kusaka ${ }^{1}$, \\ Nobuyasu Yamamoto ${ }^{1}$, Kenichi Tsujita ${ }^{2}$ \\ ${ }^{1}$ Department of Cardiovascular Center, Miyazaki Prefectural Nobeoka Hospital, Nobeoka, Japan \\ ${ }^{2}$ Department of Cardiovascular Medicine, Graduate School of Medical Sciences, \\ Kumamoto University, Kumamoto, Japan
}

Three-dimensional (3D) optical coherence tomography (OCT) (St. Jude Medical, St. Paul, MN)-guided coronary stenting was performed for stenosis at the bifurcation of the left anterior descending artery (LAD) and diagonal (DG) branch. A coronary angiography revealed true bifurcation lesion with Medina classification (1,1,1) (Fig. 1A). After lesion modification with rotational atherectomy and kissing balloon inflation (KBI) technique, crossover stenting with a Resolute Onyx zotarolimus-eluting stent $(2.25 \times 30 \mathrm{~mm}$, Medtronic Cardiovascular, Santa Rosa, CA) was done in the LAD lesion (Fig. 1B). A guidewire was recrossed to the DG branch following the proximal optimization technique with a $2.25 \times 8 \mathrm{~mm}$ noncompliant balloon. Post-rewired $3 \mathrm{D}-\mathrm{OCT}$ imaging visualized quite small distal cell and recrossed guidewire position at the proximal larger cell (Fig. 1C, D). After confirming proximal rewiring by $3 \mathrm{D}-\mathrm{OCT}$ imaging, rewiring the distal cell was attempted. This option was not selected as the distal cell was quite small and it was decided to perform KBI in the proximal cell. As therewrapped $2.0 \times 12 \mathrm{~mm}$ noncompliant balloon used in pre-stenting KBI could not be advanced to the DG branch, presumably because of the jailed strut, using $2.25 \mathrm{~mm}$ noncompliant balloon located in the distal main vessel as an anchor balloon, the balloon was finally pushed into the DG branch (Fig. 1E). Final angiography after post-stenting KBI $(2.25 \times$ $\times 8 \mathrm{~mm}$ to LAD and $2.0 \times 12 \mathrm{~mm}$ to DG branch) showed favorable findings with optimal dilatation of both LAD/DG branch lesions (Fig. 1F). A final 3D-OCT image pullback from the LAD revealed excellent stent expansion and disappearance of the jailed strut at the DG orifice (Fig. 1G), and 3D-OCT pullback from the DG branch clearly imaged good apposition of the strut folded toward the DG branch at a sharp angle covering the LAD/DG carina (Fig. $1 \mathrm{H})$.

In bifurcation stenting, 3D-OCT imaging enables visualization of the link of stent struts and also to control the recrossing point of the side-branch guidewire. It has been demonstrated that distal rewiring resulted in favorable stent positioning against the side branch ostium leading to a reduction of incomplete stent apposition and potentially better clinical outcomes in the free carina type. As the jailed strut may remain at the carina even after KBI when recrossing the "proximal" cell, "balloon pushfold" method with proximal cell guidewire crossing has been proposed to invert the strut toward the side branch in this situation. In the current case, it was speculated that pushing the rewrapped balloon with larger crossing profile combined with the "Anchor balloon technique" folded the jailing strut, and final KBI appose it to the side-branch-sided carina. Furthermore, the attachment of struts on the distal main vessel by anchor balloon may prevent stent deformation and relate to folding of the jailing strut to the side branch. 3D-OCT-based guidewire recrossing strategy can help the development of optimal bifurcation stenting strategy.

Conflict of interest: Dr. Kenichi Tsujita has received grants from Goodman Co., Ltd. All the other authors have nothing to disclose.

Address for correspondence: Kenichi Tsujita, MD, PhD, FJCC, FJCS, FESC, FACC, Department of Cardiovascular Medicine, Graduate School of Medical Sciences, Kumamoto University, 1-1-1, Honjo, Chuo-ku, Kumamoto, 860-8556, Japan, tel: +81-96-373-5175, fax: +81-96-362-3256, e-mail: tsujita@kumamoto-u.ac.jp 


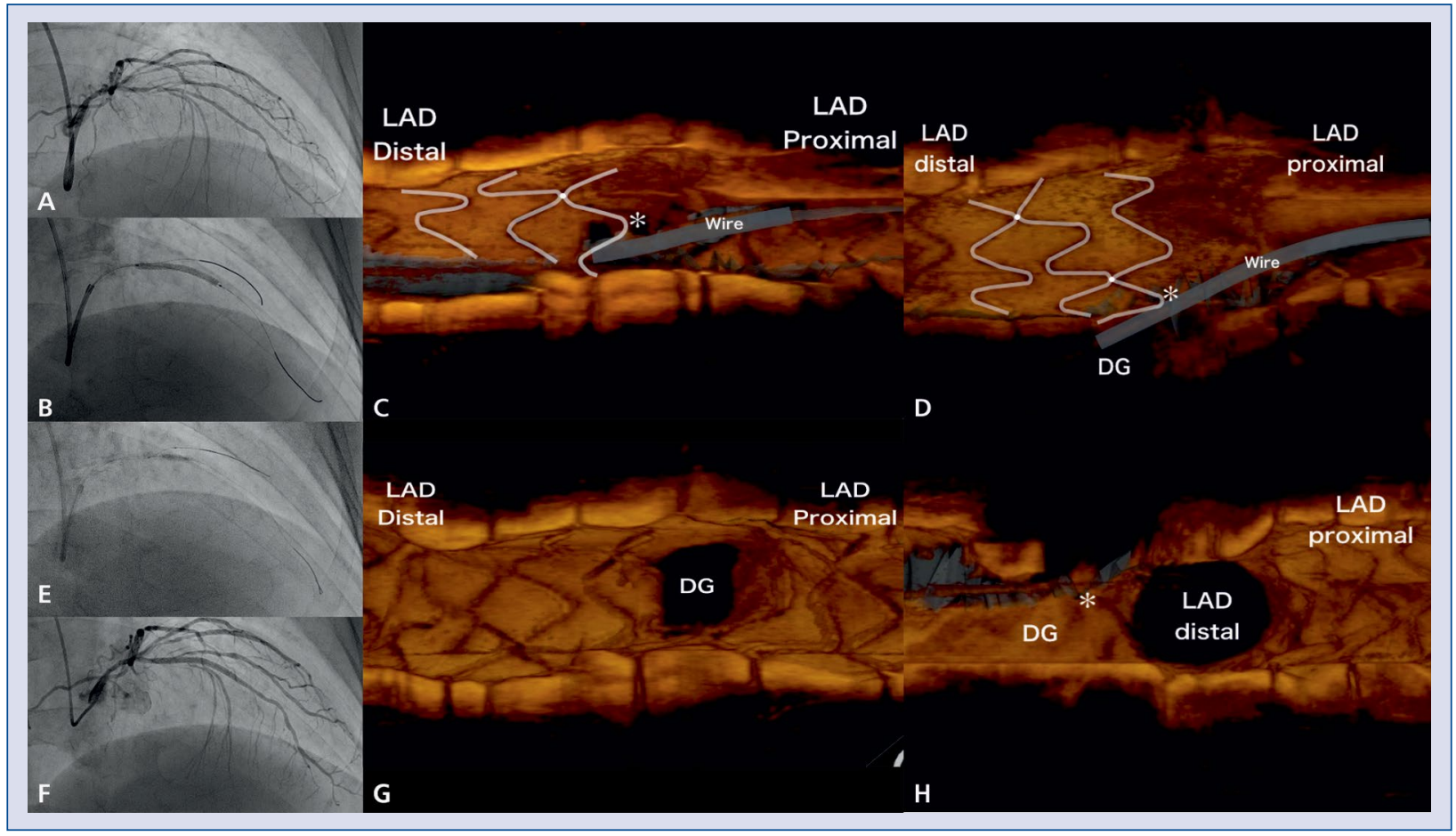

Figure 1. Coronary angiography and three-dimensional (3D) optical coherence tomography (OCT) findings. Initial coronary angiography (CAG) (A) showed significant stenoses at the bifurcation of the left anterior descending artery (LAD) and the diagonal (DG) branch with a Medina classification $(1,1,1)$. After LAD main branch stenting (B), poststenting 3D-OCT pullback from LAD (C. Frontal view; D. Lateral view) visualized existence of jailed stent strut $\left(^{*}\right)$. Following rewrapped balloon delivery to DG branch with the anchor balloon technique (E), final CAG confirmed optimal angiographic findings $(\mathbf{F})$. Final 3D-OCT pullback from LAD $(\mathbf{G})$ and DG branch $(\mathbf{H})$ revealed favorable folding of jailed strut toward DG branch. 